\title{
Cystic Lymphangioma of Pancreas
}

\author{
Chhagan Bihari ${ }^{1} \cdot$ Archana Rastogi $^{1} \cdot$ S. Rajesh $^{2}$. \\ Ankur Arora $^{2} \cdot$ Asit Arora $^{3}$ • Nikhil Kumar ${ }^{3}$
}

Received: 24 March 2014 / Accepted: 14 April 2015 /Published online: 15 May 2015

(C) Indian Association of Surgical Oncology 2015

\begin{abstract}
Lymphangiomas are benign hamartomatous malformations which can arise either from congenitally sequestered lymphatic channels or due to acquired obstruction caused by fibrosis of lymph channels. They are common in the pediatric age group in the soft tissue of neck and the axilla. Abdominal lymphangiomas are rare; even rarer is the primary involvement of pancreas. It occurs more frequently in females and is often located in the distal pancreas. The authors report the case of cystic lymphangioma of pancreas in a 26-year old female presenting with recurrent episodes of upper abdominal pain that was treated with laparoscopic cyst excision. Although exceptionally rare, lymphangioma of the pancreas should be considered in the differential diagnosis of pancreatic cystic lesions, especially in young women.
\end{abstract}

Keywords Cystic lymphangioma $\cdot$ Pancreas $\cdot$ Cyst excision

\section{Introduction}

Lymphangiomas are benign cystic lesions that occur as a result of congenital or acquired obstruction of lymphatic flow leading to development of lymphangiectasia $[1,2]$. Histologically, they

S. Rajesh

rajesh387@gmail.com

1 Department of Pathology, Institute of Liver and Biliary Sciences, New Delhi 110070, India

2 Department of Radiology, Institute of Liver and Biliary Sciences, D1, Vasant Kunj, Off Abdul Gaffar Khan Marg, New Delhi 110070, India

3 Department of Hepatobiliary Surgery, Institute of Liver and Biliary Sciences, New Delhi 110070, India are composed of dilated cystic spaces containing proteinaceous eosinophilic fluid, separated by fine septa and lined with endothelial cells [2]. They are most commonly seen in the neck and axilla $[1,2]$. Abdominal lymphangiomas are rare; even rarer is the primary involvement of pancreas, accounting for less than one percent of reported cases [2]. It was first identified in 1913 by Koch as a form of benign cyst secondary to blocked regional lymphatic ducts [3]. Since then, fewer than 60 such cases have been reported in the English medical literature [4]. Patients with pancreatic lymphangiomas may be asymptomatic or may present with abdominal distension or a palpable mass. More acute cases are reported in children whereas chronic development is common among adults [5]. The typical appearance on imaging is a lobulated complex cystic mass with septations [6]. We herein report the case of a young female with cystic pancreatic lymphangioma and review the relevant literature.

\section{Case Report}

A 26 year old female presented to our out-patient department with complaint of recurrent upper abdominal pain for two years. There was no history of prior surgery, trauma or any other co-morbidity. Her laboratory investigations were within normal reference ranges. Ultrasound examination showed a well-defined anechoic lesion of $5 \times 5 \mathrm{~cm}$ in the left subhepatic space abutting the body of pancreas (Fig. 1). Contrastenhanced computed tomographic (CECT) scan revealed a well-defined lobulated hypodense lesion in the pre- pancreatic space arising from the pancreatic body (Fig. 2). Further, 3Dmagnetic resonance cholangiopancreatography (MRCP) revealed that the lesion was separate from the main pancreatic duct (arrowheads) (Fig. 2). Patient underwent laparoscopic pancreatic cyst excision (Fig. 3). Histopathological examination of the specimen showed several variable sized cystic 
Fig. 1 Gray-scale ultrasound (a) image shows a well-defined anechoic lesion (arrows) in the left subhepatic space while axial T2weighted fat saturated MRI image (b) demonstrates a hyperintense lesion (arrow) confirming its cystic nature

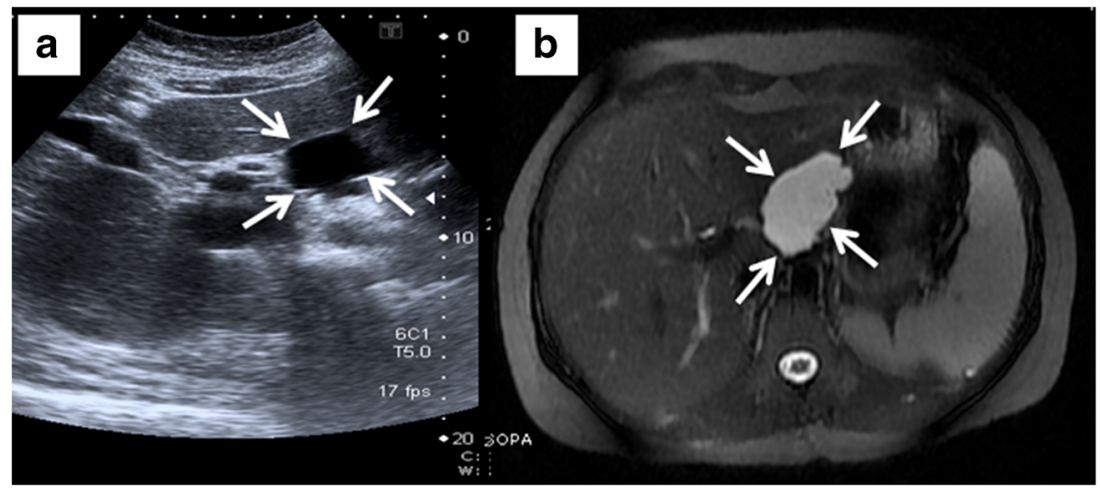

spaces comprising of dilated lymphatics vessels lined by benign flattened to cuboidal endothelial cells (Fig. 4). Small collections of lymphoid cells and lymph were noted in the lumen. On immunohistochemical evaluation these lining cells were CD31 positive (Fig. 4). Pathological diagnosis of pancreatic cystic lymphangioma was rendered. At 6 months follow-up, the patient remains asymptomatic with no clinical or radiological evidence of recurrence.

\section{Discussion}

Lymphangiomas are commonly identified in the neck and axilla at younger age group [1]. Other uncommon sites have also been described in the literature, including pleura, pericardium, mediastinum, groin, bones and abdomen [2]. Abdominal sites comprise only $1 \%$ of all lymphangiomas with mesentery and retroperitoneum (perirenal) forming the vast majority [7]. Lymphangiomas of the pancreas are even rarer accounting for $0.2 \%$ of all pancreatic neoplasms [3]. Pancreatic lymphangiomas are usually encountered in young females and pancreatic body is more commonly involved than the tail [8].

The lesion is thought to result from the abnormal development of lymphatics in the dorsal mesoduodenum during the second and third months of fetal life. This may result in obstruction of lymph flow and cystic dilatation of lymphatics. Other acquired conditions may also lead to occurrence of lymphangioma such as trauma, inflammatory process, and surgery or radiation therapy. Inadequately drained lymphatic vessels progressively dilate and form a cystic mass lesion [5].

Macroscopically, cystic lymphangiomas appear as soft, multiloculated cystic masses that contain serous, serosanguinous, or lymphatic fluid [6]. Histologically, pancreatic lymphangiomas are multiloculated cystic tumors composed of variably sized, dilated lymphatic channels, divided
Fig 2 Axial (a, c) and coronal reformatted (b) CECT scan images demonstrate a welldefined lobulated hypodense lesion (white arrows) in the prepancreatic space extending along the gastrohepatic ligament into the left subhepatic region. It shows a stalklike projection abutting the pancreatic body (arrowhead in image c). Pancreas (black arrow) and the main pancreatic duct (arrowhead) can be well appreciated (b). 3D-MRCP image (d) demonstrates the lesion (arrow) to be separate from the main pancreatic duct (arrowheads)

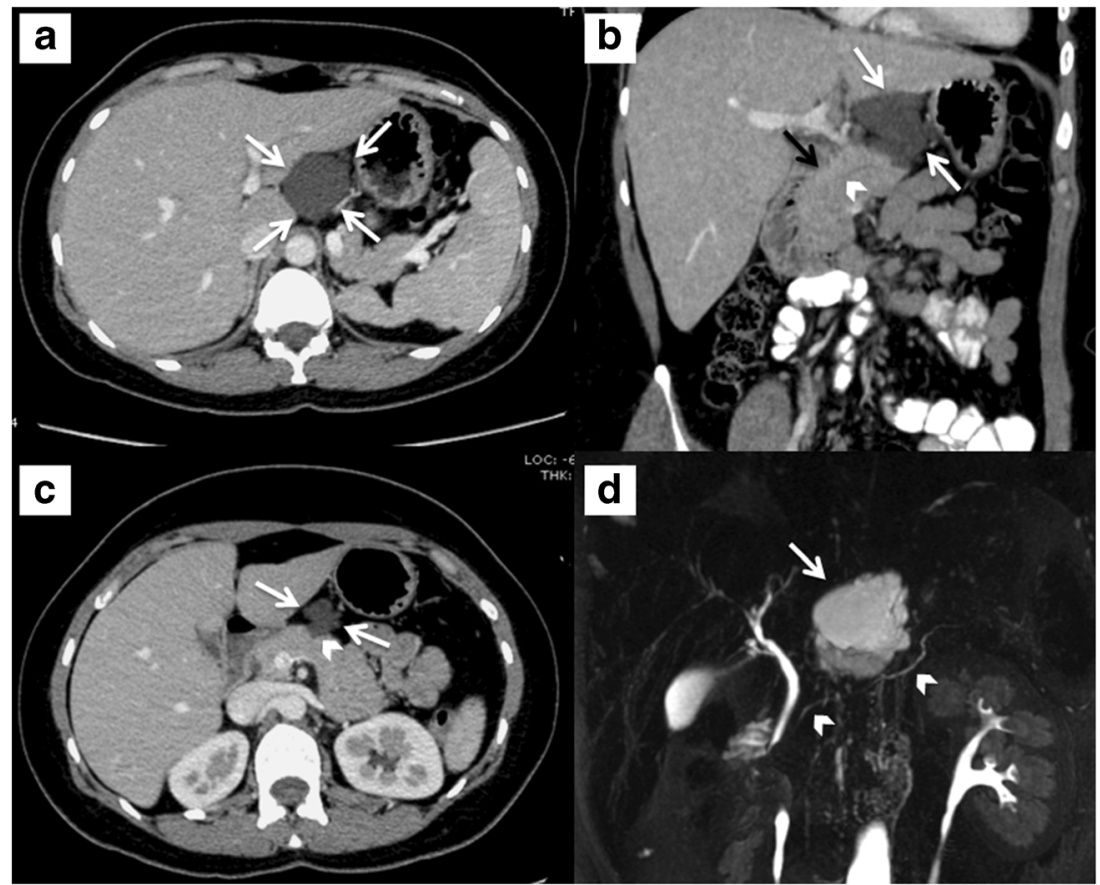




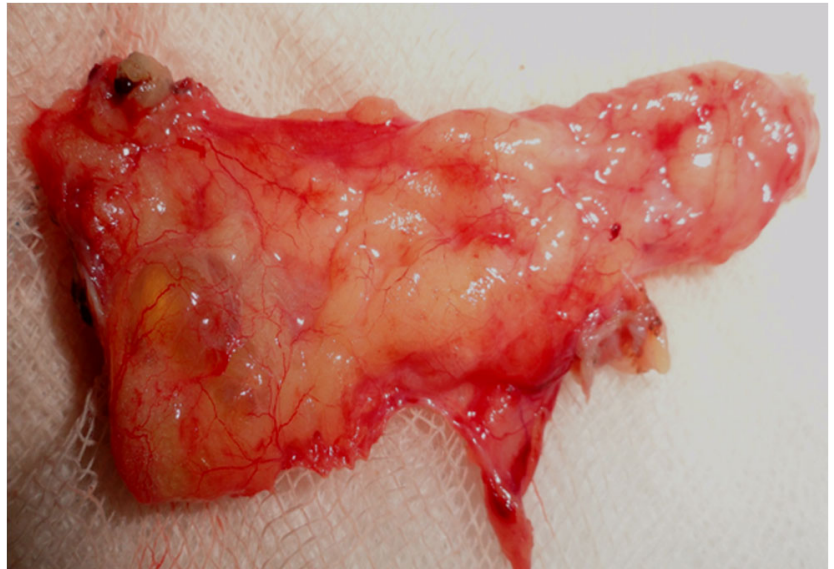

Fig 3 Resected specimen shows a lobulated cystic mass

by thin septae with fibrocollagenous stroma. These cysts are lined by endothelial cells which are usually flattened but at times they may be cuboidal or low columnar in nature. Aggregates of lymphocytes are found in the lumina and the adjacent stroma. The septa and walls contain occasional smooth muscle fascicles and various amounts of collagenous connective tissue. Three histologic types of lymphangiomas have been described: cystic, capillary, and cavernous. Only the cystic and cavernous types have been reported in the pancreas [6].

This disease entity lacks specific clinical symptoms and can grow reasonably large prior to manifestation of symptoms. The clinical presentation is inconsistent and depends on the location and size of the lesion. Patients may be symptomatic presenting with abdominal pain, nausea, vomiting and palpable abdominal mass or may be asymptomatic. The current case presented with abdominal pain. Complications like infection, haemorrhage, torsion, rupture or intestinal obstruction presenting as acute abdomen have also been described [9].

Sonography shows a well-defined, anechoic, multiseptated lesion in the pancreatic region. CT usually demonstrates homogeneous cystic mass usually with multiple fine septations and thin walls that may enhance after intravenous contrast administration. Rarely, phlebolith-like calcifications may be present. On MRI, the cystic spaces appear hypointense on T1-weighted images and hyperintense on T2- weighted images [6]. The differential diagnosis includes pseudocysts, and cystic pancreatic neoplasms. Pseudocysts are unilocular usually without septations and may be associated with pancreatic duct dilatation [9]. In addition, attendant clinical or imaging features of pancreatitis are usually demonstrable. Serous cyst adenomas may resemble cystic lymphangiomas in being multiloculated but a fibrous central scar with or without a characteristic stellate pattern of calcification (seen in up to $30 \%$ of cases) is considered highly specific and virtually pathognomonic for serous cystadenoma. Mucinous cyst adenomas are usually larger oligocystic masses that may contain solid enhancing tissue and occasional peripheral egg-shell calcification which help in their differentiation from lymphangiomas [9]. Intraductal papillary mucinous tumors usually show ductal communication and/or dilatation. In the current case, the tumour was a lobulated well circumscribed cystic mass without calcification, enhancing solid component or ductal communication.

Endoscopic ultrasound guided aspirates with high triglycerides and presence of lymphocytes can suggest the diagnosis [10]. The definitive diagnosis of pancreatic lymphangioma, however, can be made only by excision and histopathologic
Fig 4 Cystic spaces (arrows) and pancreatic tissue (thin arrow) (a). Flattened endothelial lining of the cyst(arrow) (b). Cystic lumen containing mature lymphocytes (arrow) and lymph (thin arrow) (c) $[\mathrm{HE}, 200 \times] . \mathrm{CD} 31$ positive lining endothelial cells (d)
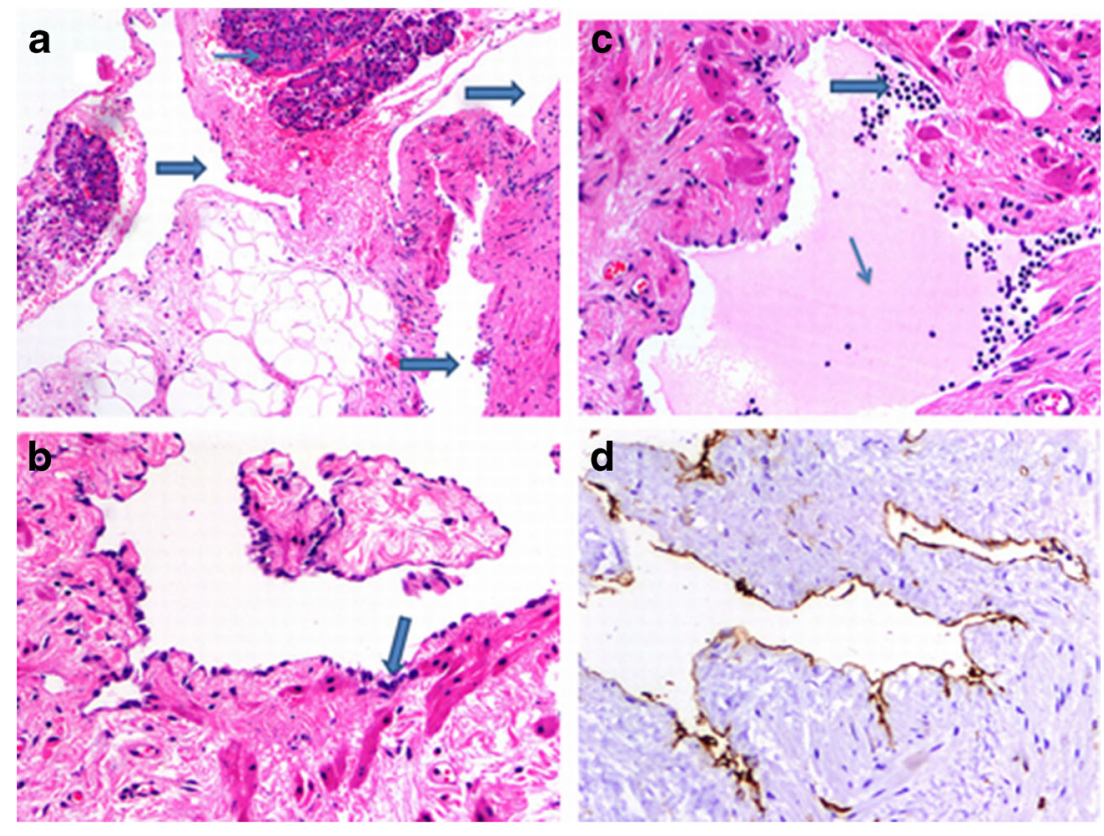
examination. Surgical excision is usually curative [3]. Incomplete excision is likely to lead to a local relapse [6].

In conclusion, cystic lymphangiomas, although rare, should be kept as a differential diagnosis while assessing a pancreatic cystic lesion, especially in young females.

Conflict of Interest All authors state that there are no conflicts of interests.

\section{References}

1. Fahimi H, Faridi M, Gholamin S, Molanaee S, Khorsandi M (2010) Cystic lymphangioma of the pancreas: diagnostic and therapeutic challenges. JOP 11(6):617-9

2. Colovic RB, Grubor NM, Micev MT, Atkinson HD, Rankovic VI, Jagodic MM (2008) Cystic lymphangioma of the pancreas. World J Gastroenterol 14:6873-5
3. Paal E, Thompson LD, Heffess CS (1998) A clinicopathologic and immunohistochemical study of ten pancreatic lymphangiomas and a review of the literature. Cancer 82:2150-2158

4. Sohn BK, Cho CH, Chae HD (2011) Cystic lymphangioma of the pancreas. J Korean Surg Soc 81(2):141-5

5. Losanoff JE, Richman BW, El-Sherif A, Rider KD, Jones JW (2003) Mesenteric cystic lymphangioma. J Am Coll Surg 196: 598-603

6. Koenig TR, Loyer EM, Whitman GJ, Raymond AK, Charnsangavej C (2001) Cystic lymphangioma of the pancreas. AJR Am J Roentgenol 177(5):1090

7. Abe H, Kubota K, Noie T, Bandai Y, Makuuchi M (1997) Cystic lymphangioma of the pancreas: a case report with special reference to embryological development. Am J Gastroenterol 92:1566-1567

8. Khandelwal M, Lichtenstein GR, Morris JB, Furth EE, Long WB (1995) Abdominal lymphangioma masquerading as a pancreatic cystic neoplasm. J Clin Gastroenterol 20(2):142-4

9. Afzal S, Masroor I, Shafqat G (2014) Pancreatic lymphangioma. J Coll Physicians Surg Pak 24(1):60-1

10. Coe AW, Evans J, Conway J (2012) Pancreas cystic lymphangioma diagnosed with EUS-FNA. JOP 13(3):282-284 